\title{
Téoros
}

Revue de recherche en tourisme

\section{La promotion d'une destination auprès de la clientèle gaie} L’expérience du Québec

\section{Jean Bouffard et Fair Gordon}

Volume 19, numéro 2, été 2000

Le tourisme des gais et des lesbiennes

URI : https://id.erudit.org/iderudit/1071966ar

DOI : https://doi.org/10.7202/1071966ar

Aller au sommaire du numéro

Éditeur(s)

Université du Québec à Montréal

ISSN

0712-8657 (imprimé)

1923-2705 (numérique)

Découvrir la revue

Citer cet article

Bouffard, J. \& Gordon, F. (2000). La promotion d'une destination auprès de la clientèle gaie : l'expérience du Québec. Téoros, 19(2), 53-55.

https://doi.org/10.7202/1071966ar d'utilisation que vous pouvez consulter en ligne.

https://apropos.erudit.org/fr/usagers/politique-dutilisation/ 


\section{Jean Bouffard et Fair Gordon}

Les plus récentes recherches de Community Marketing, société spécialisée dans le tourisme gai basée à San Francisco, démontrent que la clientèle gaie nord-américaine voyage cinq fois plus que les hétérosexuels et dépense davantage au cours de ces voyages. Le fait que $75 \%$ de ces 16 millions de clients potentiels aient un revenu familial plus élevé que la moyenne ne laisse aucun doute sur l'importance de ce marché pour l'industrie touristique. Cela dit, nous tracerons d'abord un bref historique de l'intérêt et des démarches de promotion de Tourisme Québec et de ses partenaires auprès du marché gai. Nous établirons ensuite une typologie sommaire des différentes strates de ce segment de marché et nous traiterons de l'image du Québec auprès de ces clientèles. Finalement, nous traiterons de l'apport de la clientèle gaie au positionnement général de la destination et suggérerons diverses avenues de promotion et de mise en marché de la destination auprès de ce segment.

\section{PETIT HISTORIQUE DE L'INTÉRÊT DE TOURISME QUÉBEC POUR LA CLIENTÈLE GAIE}

Comme cela se passe souvent dans l'industrie du tourisme, des destinations se trouvent des marchés alors que les marchés ont déjà découvert des destinations. Montréal et Québec — en fait, le Québec en général - ont toujours eu une approche tolérante de la différence. C'est probablement pour cette raison, en plus de la vitalité de la communauté gaie locale, que se sont développés une infrastructure et des événements qui se prêtent au tourisme gai et une tolérance générale de la clientèle gaie au sein même de la destination : établissements de tourisme grand public (clientèles diverses), la rue, attitude des gens qui travaillent dans les services. Nous reviendrons sur cette typologie clientèle gaie versus tourisme gai, mais d'abord faisons un survol de la façon dont Tourisme Québec a été mis en présence d'un réseau. Tout comme un coup de foudre, il s'est agi d'une rencontre inattendue, mais contrairement au coup de foudre, c'est une relation qui a tout pour durer.

Au printemps 1994, notre bureau de New York prenait connaissance de l'existence de salons à l'intention d'agents de voyages s'intéressant aux destinations gay friendly. Ne disposant à l'époque que de peu de données sur cette clientèle en particulier, nous avons alors convenu de faire de la recherche action et de participer au seul salon de l'époque à l'intention des clientèles gaies sur la côte est. Community Marketing, promoteur de ce salon, en organisait alors deux, le second sur la côte ouest à San Francisco.

Depuis 1995, nous participons non seulement au salon de Community Marketing de New York, mais aussi à celui de San Francisco ainsi qu'aux nouveaux salons d'Atlanta et de Chicago. L'importance du marché et la position privilégiée du Québec devenant de plus en plus évidentes, notre bureau de Los Angeles a entamé des pour- parlers avec Community Marketing pour accroître et diversifier les efforts auprès de cette clientèle. C'est ainsi qu' au printemps 1997 était lancée, à titre d'essai, la campagne Visit Gay Friendly Québec qui englobait, en plus de la participation aux salons pour agents de voyages dont Community Marketing était maître d'œuvre, la réalisation et la distribution d'une affiche positionnant la destination, le développement d'un site Internet sur le même thème et des activités de relations de presse.

Cette première collaboration ayant été un succès, nous avons reporté pour l'exercice 1998-1999 notre entente avec Community Marketing en ajoutant un nouvel élément à la stratégie : l'élément produit. Tourisme Montréal et l'Office du Tourisme et des Congrès de la Communauté urbaine de Québec sont alors devenus partenaires. La manifestation la plus visible de ce nouvel élément a pris la forme d'un dépliant réalisé avec la collaboration d'Air Canada et mettant en vedette le produit de quatre voyagistes gay friendly à destination du Québec. Bien que ce volet n'ait pas eu le succès escompté, il a permis d'en apprendre davantage sur le comportement d'achat de la clientèle et a contribué à confirmer et à renforcer l'engagement du Québec comme destination touristique auprès de la clientèle gaie.

Parallèlement, Montréal obtenait en 1998 le congrès annuel de l'IGLTA (International Gay and Lesbian Travel Association) qui s'est révélé un grand succès et, de mémoire de congressiste, le meilleur que l'IGLTA ait tenu à ce jour. En outre, les partenaires locaux et régionaux intéressés 
à ce segment de marché ont commencé à produire des documents promotionnels adaptés à la clientèle gaie.

Cela allait porter fruit dès l'année suivante, Tourisme Québec étant invité à prendre part aux salons Sizzling Cities, circuit de séminaires visant les agents de voyages et les voyagistes qui s'intéressent au tourisme gai. Ces séminaires ont lieu dans six grandes villes américaines et sont organisés conjointement par les bureaux de tourisme participants, soit la British Tourist Authority, la Maison de la France, le New South Wales Tourism Office et l'Office national de Tourisme allemand. Dans les faits, cette invitation constituait la reconnaissance du Québec par ses pairs comme une des principales destinations gaies de la planète. À sa troisième année d'existence, Sizzling Cities s'impose déjà comme l'activité de promotion par excellence des destinations internationales auprès du réseau de distribution gai américain.

Pour l'exercice 1999-2000, la troisième année de notre entente avec Community Marketing, il a été convenu de délaisser le volet produit au profit des relations de presse. En 1998, 1999 et au début de 1'an 2000 , la presse touristique gaie a accepté l'invitation de Tourisme Québec à participer à diverses tournées de presse, notamment dans Montréal, Québec, Charlevoix et d'autres régions. Ces tournées ont généré une couverture médiatique importante et fort positive pour le Québec, tant en Amérique du Nord qu'en Europe. Tourisme Québec et ses partenaires sont très satisfaits des résultats obtenus à ce jour et en sont présentement à évaluer les éléments sur lesquels ils mettront 1'accent pour l'exercice 2000-2001.

Le choix des relations de presse s'explique par le fait que la clientèle gaie est une clientèle avertie qui maîtrise la plupart des moyens modernes de se procurer l'information dont elle a besoin et qu'il est plus profitable de positionner la destination et de la garder à la mémoire du consommateur potentiel plutôt qu'essayer de mettre au point des formules « tout compris » que l'on mettrait en marché au moyen des réseaux traditionnels. De même, le « net » constitue un médium que la clientèle gaie maîtrise exceptionnellement bien et dont elle fait largement usage.

Tourisme Québec maintiendra sa participation à Sizzling Cities et à une série de scènes de vente aux États-Unis pour le marché gai gérées par Travel Alternatives Group. Nous serons encore présents au congrès de l'IGLTA et comptons soutenir la candidature de Tourisme Montréal pour les Gay Games. Tourisme Québec compte également offrir, en collaboration avec ses partenaires, des séminaires de formation sur la clientèle gaie à l'intention des fournisseurs de services touristiques du Québec intéressés par ce segment de marché.

\section{Clientèle Gaie VERSUS TOURISME GAI}

Pour les fins de notre propos, nous n'établirons qu'une typologie sommaire. Nous distinguerons ici clientèle gaie qui a rapport à l'individu et tourisme gai qui a plutôt trait à l'infrastructure, c'est-à-dire aux équipements (hôtels, centres de villégiature, bars, etc.) et aux événements. La clientèle gaie a toujours existé, existera toujours et n'a pas attendu l'avènement d'établissements s'affichant comme gais ou gay friendly ou encore la tenue d'événements s'adressant avant tout à la clientèle gaie.

Le Québec a toujours été une société tolérante. Bien que plusieurs identifient la «Révolution tranquille » comme l'événement ayant donné le coup de grâce à nos démons officiels, Montréal a toujours eu la réputation de ne pas se formaliser des comportements condamnés par la bourgeoisie de l'époque ou le vieil héritage victorien du XIX ${ }^{\mathrm{e}}$ siècle. Montréal la tolérante, Montréal et son red light, Montréal la ville qui aime célébrer. Le Québec et Montréal plus particulièrement ont probablement toujours été des destinations prisées par la clientèle gaie. La plus grande et aussi la plus cosmopolite des villes du Québec, Montréal constituait d'emblée un terreau des plus fertiles pour l'apparition d'une communauté gaie locale structurée et l'organisation d'un tourisme gai avec ses infrastructures propres. Ces infrastructures elles-mêmes sont le résultat du travail d'organisation d'une communauté gaie locale créative et persévérante.

Montréal a mis sur pied un grand nombre d'événements qui s'adressent spécifiquement à ce marché. Québec n'est d'ailleurs pas en reste avec son Fest'Iver qui a débuté à l'hiver 1998, mais qui a marqué une pause en 2000. Mont-Tremblant également a déjà quelques événements à son actif.

\section{UNE IMAGE POSITIVE DE LA DESTINATION}

L'adoption de la Loi 32 par le Gouvernement du Québec le 10 juin 1999, par un vote unanime de l'Assemblée Nationale, a confirmé et renforcé la perception, par la communauté gaie américaine, du Québec comme chef de file en matière de reconnaissance des droits des gaies et des lesbiennes. Sous la bannière « Le sexe n'a plus d'importance », la Loi 32 reconnaît que les conjoints de même sexe sont considérés tels des conjoints de fait ; ils ont les mêmes droits, les mêmes privilèges et les mêmes obligations. Cet aspect de la législation québécoise est très important pour la communauté gaie américaine et tient presque lieu de symbole car celle-ci fait encore face à beaucoup d'obstacles sociaux et nombre de barrières politiques pour se faire reconnaitre les mêmes droits.

Air Canada a par ailleurs été l'un des premiers transporteurs au monde, sinon le premier, à reconnaître la clientèle gaie tel un segment de marché spécifique et à participer activement à des démarches promotionnelles visant spécifiquement cette clientèle. On lit aussi à maintes reprises dans la presse gaie que le Gouvernement du Québec, par le biais de Tourisme Québec, a été le premier gouvernement à se promouvoir officiellement et activement auprès de la population gaie mondiale. Tourisme Québec a d'ailleurs soutenu financièrement certains festivals qui placent aujourd'hui Montréal dans le peloton de tête des villes gay friendly à l'échelle internationale. La clientèle gaie reçoit un bon niveau d'acceptation de nos jours, mais tel n'a pas toujours été le cas. La situation actuelle est le résultat des quarante dernières années d'efforts des milieux militants et chaque parcelle de reconnaissance a été gagnée de chaude lutte. Ajoutons que c'est une société solidaire qui sait reconnaître ses supporters.

\section{LES CLIENTÈLES GAIES : DES CLIENTĖLES DE TREND.SETTERS}

La clientèle gaie constitue une clientèle de prédilection. Les ménages gais ont typiquement un revenu disponible plus élevé que la moyenne. Les gais et les lesbiennes ont aussi une propension au voyage plus élevée que la moyenne. Dès le départ, il s'agit d'une clientèle très lucrative pour 
une destination car elle sort, va au restaurant, au bar et a une propension à utiliser le réseau d'hébergement commercial plus élevée que la moyenne.

La clientèle gaie est aussi créatrice de tendances en termes de popularité de destination et son champ d'influence déborde bien la communauté gaie, que ce soit par de vastes réseaux d'ami(e)s ou par ses relations professionnelles dans tous les milieux. Les exemples sont nombreux où des destinations ignorées par le tourisme de masse sont devenues populaires grâce à l'attention des clientèles gaies.

\section{CONCLUSION : UN MARCHÉ DIVERSIFIÉ ET EN MOUVANCE CONSTANTE}

Notre expérience du marché gai nous a démontré l'importance de ce segment de marché qui, comme grand nombre de segments, n'est pas monolithique, c'està-dire que différents produits (ex : événements versus séjour) ont la préférence de sous-segments distincts, mais peuvent parfois se croiser. Ces sous-segments achètent le produit par le biais de divers réseaux, selon la nature du produit ou encore selon les habitudes de consommation. Ainsi, on aura tendance à faire appel aux services d'un voyagiste quand il s'agira d'assister à un événement majeur sur le circuit international des fêtes gaies ; pour un séjour urbain ou de villégiature, on achètera plutôt directement ou encore d'un agent en qui l'on a confiance et qui connaît les fournisseurs gay friendly dans les diverses destinations.

Au plan des communications, notons que la clientèle gaie à une grande maîtrise d'Internet. Ce véhicule se prêtant très bien aux produits visant des créneaux bien ciblés de clientèle, il constitue un médium de prédilection pour l'entreprise qui s'intéresse à la clientèle gaie. Par ailleurs, comme le milieu gai lit à la fois la presse traditionnelle et la presse gaie, une démarche soutenue de relations de presse constitue également un excellent investissement pour qui s'intéresse à ce marché. Nonobstant ce qui précède, il ne faut pas oublier que le marché gai, comme tous les autres marchés est en mouvance et qu'il faut constamment adapter son marketing mix pour maximiser son dollar promotionnel.

En outre, il faut toujours garder à l'esprit que la clientèle gaie ne portera pas son

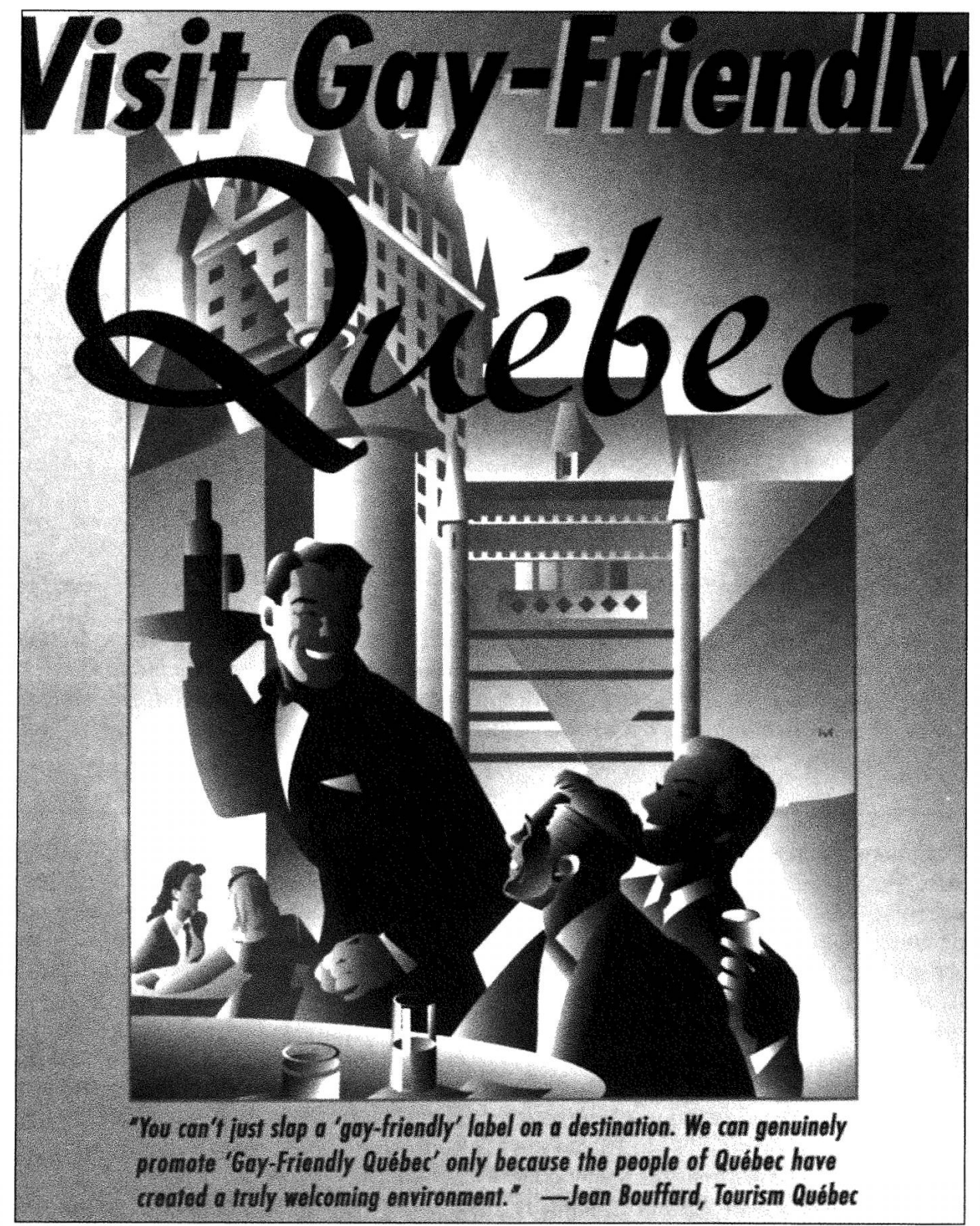

Publicité de Tourisme Québec destinée au marché étasunien gai et lesbien.

intérêt sur une destination sans potentiel. Aussi, être reconnue comme destination gay friendly constitue beaucoup plus le constat d'une situation effective qu'une étiquette sur une image que l'on veut se donner. Une destination est gay friendly ou ne l'est pas. Le Québec possède tous les atouts lui valant l'attrait de ce segment de marché. Il nous reste maintenant, tous ensemble, à soutenir l'intérêt et à maintenir la qualité du produit et à poursuivre l'élargissement de notre réseau de partenaires.

Jean Bouffard est chef de pupitre pour les ÉtatsUnis et le Canada à la Direction du Développement des marchés de Tourisme Québec. Il encadre le travail des effectifs de représentation commerciale de Tourisme Québec sur ces marchés. Conseillant l'organisation quant à la promotion de la destination, il développe et gère les activités liées à la distribution du produit à ces mêmes marchés.

Fair Gordon est directrice régionale de Tourisme Québec pour l'Ouest des États-Unis, à Los Angeles. Elle fut associée aux efforts de Tourisme Québec auprès du marché gai dès le début. Ayant développé l'entente de mise en marché conclue avec Community Marketing, elle veille toujours à son suivi. Elle participe activement aux activités de représentation auprès du marché gai, notamment au congrès annuel de l'IGLTA et à Sizzling Cities.

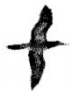

